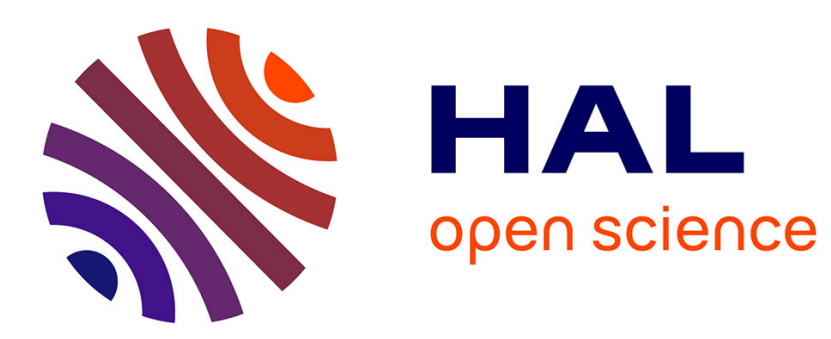

\title{
A framework for the design of rotating Multiple Tuned Mass Damper
}

Kévin Jaboviste, Emeline Sadoulet, Olivier Sauvage, Gael Chevallier

\section{To cite this version:}

Kévin Jaboviste, Emeline Sadoulet, Olivier Sauvage, Gael Chevallier. A framework for the design of rotating Multiple Tuned Mass Damper. Conference and Exposition on Structural Dynamics, Feb 2020, Houston, United States. hal-02993955

\section{HAL Id: hal-02993955 \\ https://hal.science/hal-02993955}

Submitted on 7 Nov 2020

HAL is a multi-disciplinary open access archive for the deposit and dissemination of scientific research documents, whether they are published or not. The documents may come from teaching and research institutions in France or abroad, or from public or private research centers.
L'archive ouverte pluridisciplinaire HAL, est destinée au dépôt et à la diffusion de documents scientifiques de niveau recherche, publiés ou non, émanant des établissements d'enseignement et de recherche français ou étrangers, des laboratoires publics ou privés. 


\title{
A framework for the design of rotating Multiple Tuned Mass Damper
}

\author{
K.Jaboviste $^{1}$, E.Sadoulet-Reboul ${ }^{1}$, O.Sauvage ${ }^{2}$, G.Chevallier ${ }^{1}$ \\ ${ }^{1}$ Univ. Bourgogne Franche-Comté FEMTO-ST Institute \\ Department of Applied Mechanics, 24, rue de l'épitaphe, F-25000 Besançon \\ ${ }^{2}$ Groupe PSA Scientific and Future Technologies Departement, Route de Gisy, F-78140 \\ Vélizy-Villacoublay \\ e-mail: kevin.jaboviste@univ-fcomte.fr
}

\begin{abstract}
The acoustic and vibratory analysis represent an essential research axis in the automotive industry because these phenomena directly affect the appreciation of the customer when using a vehicle. Indeed, the combustion engine represents the main source of mechanical energy but it generates an acyclic torque because of the explosions. This acyclism is responsible for noise and vibration fatigue. To reduce NVH issues, a possible mean is to use a Multiple Tuned Mass Damper (MTMD) adapted to rotating machine.

The purpose of this paper is to propose a framework for the design of this type of MTMD. To achieve this goal, an optimization strategy is implemented in the non-rotating and rotating case to determine the optimal distribution of the MTMD frequencies. It is based on the minimization of the elastic strain energy of the transmission chain near the torsion mode. In addition, a dedicated reduced order method is proposed to reduce the calculation costs link to the modeling of the system by the finite element method and the optimization process. However, conventional reduction order methods are not suitable for this type of problem where the shape of the modes of the main structure and the MTMD are varying according to the rotation speed. To overcome this problem, a multi-model approach is employed. Finally, the influence of the number of absorbers composing the MTMD as well as the structural damping of the absorbers and their mass on the performance of the optimal solution is presented in the non-rotating and rotating case.
\end{abstract}

Keywords : Rotating frame, Multiple Tuned Mass Damper, Optimization, Model order reduction, Passive damping

\section{Introduction}

Nowadays, the noise generated by the vehicle operation is one of the important criteria taken into account by car manufacturers. Thus, many efforts have been made for several years to reduce the noise pollution produced by the various components of the vehicle. The overall goal is to meet environmental standards that become more severe, but also to improve the comfort of passengers within the vehicle. In most of the vehicle, the motor is the main source of mechanical energy and noises. These noises are generated by the presence of motor acyclism. More specifically, this acyclism generates an oscillation of the speed of rotation of the crankshaft around a given speed which causes the occurrence of shocks and noise. Hence, to reduce NVH issues, it is possible to use Multiple Tuned Mass Damper (MTMD) adapted to rotating machines.

The use of MTMD consisting in a network of TMDs with natural frequencies distributed around the frequency to control is a strategy to absorb vibration on a frequency range around this frequency of interest. The different possible distributions for the eigenfrequencies of the MTMD have been the subject of numerous studies. Many studies consider multiple absorbers with equally spaced eigenfrequencies $[1,7,8,20,21]$. The performance of MTMD with uniformly and linearly distributed masses are compared in $[9,16]$. Optimization methodologies have been used to increase the efficiency of MTMDs and do not impose a specific profile distribution. Closed-form approximations of the TMD parameters are proposed using an integral form of the impedance in [7], optimal design theories are investigated as the minimax optimization in [22], a specific optimal design theory is proposed in [11], and a gradient-based method with unconstrained variables is used in [12]. It appears through all these studies that optimal frequency distribution can be found to ensure the vibration control. Moreover, one can note that a particular frequency distribution characterized by a high modal density around the natural frequency of the master structure allows to absorb nearly irreversibly the energy of the master structure $[3,17]$. 
On the other hand, the concept of absorber generally dedicated to rotating structures is commonly called order-tuned absorbers in the literature and allows, rather than being fixed to one and only frequency, to follow a particular motor rotation order and to be effective for a whole speed range. This particular tuning is achieved by exploiting the centrifugal force field [5] and this type of absorber has had some success in the field of rotating machines with applications on aircraft engines [10], helicopter rotors [19], camshafts [4] and car engines [13]. In 2005, Shaw and Pierre propose, in [18], an analytic study of the dynamic behavior of a flexible structure with 1 degree of freedom to which is fixed an order-tuned impact absorber. In the same year, Olson, Shaw and Pierre [15] improve this work by generalizing them in the case of cyclic symmetric structures such as bladed wheels.

This paper propose an optimal design methodology for a MTMD adapted to rotating machines instead of an order-tuned absorber [18]. The studied case is shown Figure 1(a) and represents a simplified mechanical transmission chain. It is composed of a main inertia fixed on a shaft link to the main frame by a pivoting link and an adapted mechanical coupling part connecting this subsystem to a smaller inertia. The mechanical coupling part is designed in such a way that the first mode of vibration of the whole system is a torsion mode (Figure 1(b)). A MTMD is used to reduce the vibration induced by the first torsion mode. The retained geometry [14] is a set of blades as shown Figure 1(d) and the MTMD is attached to the master structure at the end of the shaft (Figure 1(c)).

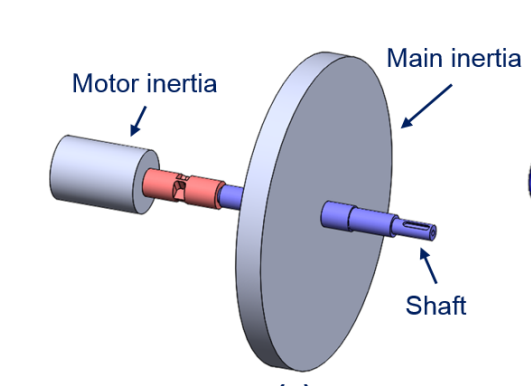

(a)
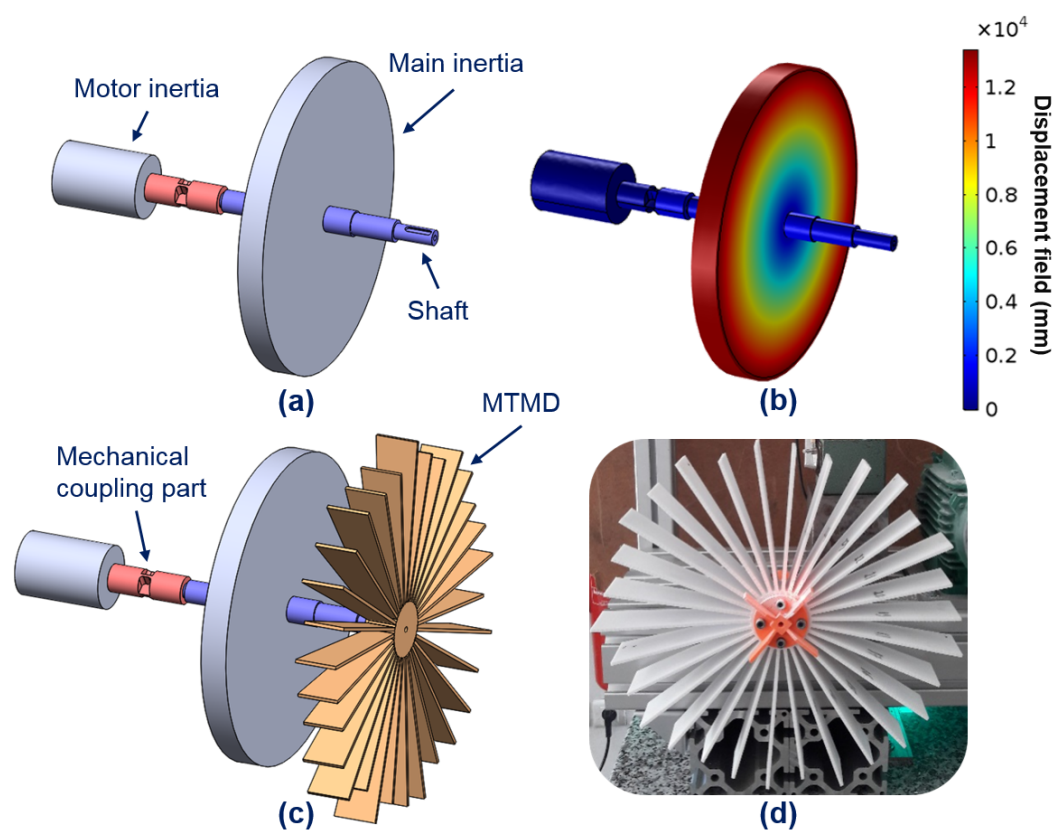

(d)

Figure 1: Studied case : CAD of the main structure (a), first torsion mode of the main structure: displacement field from FE computation (b), CAD of the main structure with the MTMD (c), and prototype of the MTMD [14] (d)

\section{Optimization of a Multiple Tuned Mass Damper adapted to rotating frame}

Modeling The Multiple Tuned Mass Damper (MTMD) attached to the main structure is composed of 30 blades and each blade behaves like a dynamic vibration absorber. The Finite Element Method is used to model the whole structure and accurately predict its physical behavior. Damping is introduced using a structural damping model for both the main structure $\left(\eta_{S}\right)$ and the MTMD $\left(\eta_{M}\right)$. In our case, the rotation speed of interest is fixed at 1500RPM and the only influencing effect is the centrifugal force which is comparable to a prechage modifying the stiffness matrices. In this context, the dynamic equation of the whole system in the frequency domain is written as,

$$
-\omega^{2} M \hat{U}+\left(K(\Omega)+K_{e}^{*}\right) \hat{U}=\hat{F}
$$

where $K_{e}^{*}$ and $M$ are respectively the complex stiffness and the real constant mass matrices of the whole structure including the MTMD, $K(\Omega)$ is a matrix that represents the influence of the centrifugal force, $\hat{U}$ is the complex displacement vector 
and $\hat{F}$ is the excitation vector. The complex stiffness is written as,

$$
K_{e}^{*}=\left(1+j \eta_{s}\right) K_{s}+\left(1+j \eta_{M}\right) K_{M}
$$

where $K_{s}$ is the stiffness matrix of the main structure and $K_{M}$ is the stiffness matrix of the MTMD. Then, to reduce computational costs and perform the optimization of the MTMD it is possible to use a projection basis. Here, the stiffness matrices vary according to the rotation speed and generate changes in MTMD eigenfrequencies and can also impact the coupling between the blades. A way to take into account these parametric variations in a model order reduction is to use a multi-model basis [2]. The multi-model approach allows to build a projection basis representative of the whole system stiffening due to the rise of rotation speed. To create this multi-model basis, three different speeds are chosen and for each of them a modal basis is extracted. It is important to choose the first speed equal to the speed of interest, here 1500RPM and the two other basis are extracted at 0 and 3000RPM. A modified Gram-Schmidt ortho-normalization [6] is used to concatenate these three basis in one multi-model basis $P$ where,

$$
P=\left[\begin{array}{lll}
\Phi^{1500} & \Phi_{\perp \Phi^{1500}}^{0} & \Phi_{\perp \Phi^{1500} \cup \Phi_{\perp \Phi^{1500}}^{0}}^{3000}
\end{array}\right]
$$

The projected operators will be noted: $m$ for the mass, $k_{s}$ for the master structure stiffness, $k_{M}$ for the MTMD stiffness and $k_{b}^{i}$ for the reduced stiffness matrix of the ith blade.

MTMD optimization at 1500RPM The design variables chosen for the MTMD optimization problem are the stiffnesses of the $N$ blades. The structural damping of the main structure and the MTMD will be considered constants. The mass and inertia of the MTMD are ten times lower than those of the master system. A coefficient $\alpha_{i}(1<i<N)$ is introduced for each blade of the MTMD such that the reduced stiffness matrix is modified as follow,

$$
k_{M}=\sum_{i=1}^{N} \alpha_{i} k_{b}^{i}
$$

Different optimization objective functions can be defined, it is chosen here to minimize the elastic strain energy on the frequency band around the resonant frequency of the torsion mode. It is defined as,

$$
\Pi(\omega)=\frac{1}{2} \hat{q}^{H}(\omega) k_{s} \hat{q}(\omega)
$$

where $k_{s}$ is the reduced elastic real stiffness matrix of the main structure at $\Omega=1500 R P M$ and $\hat{q}$ the complex generalized coordinate. $\hat{q}^{H}$ is the Hermitian transpose of $\hat{q}$. The optimization problem can thus be written as,

$$
\left\{\begin{array}{l}
\min _{\alpha_{i}} \bar{\Pi}\left(\alpha_{i}\right)=\frac{1}{\Delta \omega} \int_{\omega_{\min }}^{\omega_{\max }} \Pi(\omega) d \omega \\
-\omega^{2} m \hat{q}+\left(k(\Omega)+k_{e}^{*}\left(\alpha_{i}\right)\right) \hat{q}=\hat{f} \\
\alpha_{\text {inf }} \leqslant \alpha_{i} \leqslant \alpha_{\text {sup }}, i=1, \ldots, N
\end{array}\right.
$$

where $\alpha_{\text {inf }}$ and $\alpha_{\text {sup }}$ represent the lower and upper bounds for the parameters, $\omega_{\min }$ and $\omega_{\max }$ define the lower and upper bounds of the frequency band and $\bar{\Pi}$ is the mean value of the elastic strain energy on this frequency band.

Figure 2 presents the angular displacement $\Theta$ of the main inertia when a varying harmonic torque $T$ is applied on its lateral surface with and without MTMD. In this simulation the MTMD is composed of 30 blades and it can be observed that the MTMD significantly reduces the amplitude of the response on a wide range of frequencies around the frequency to control. One can note the existence of an optimal loss factor for the MTMD $\left(\eta_{M}=10 \%\right)$ that leads to a better attenuation efficiency. In the case of rotating machines, the rigid body mode is no longer at $0 \mathrm{~Hz}$ but higher in frequency because of the matrix $K(\Omega)$ which becomes a non-zero matrix. Moreover, as previously mentioned in the paper, the centrifugal force acts as a precharge and thus modifies the shape of the static deformation.

\section{Conclusion}

This paper proposes a methodology to determine the optimal frequency distribution for a set of blades composing a Multiple Tuned Mass Damper adapted to rotating frame in order to reduce the vibration induced by the first torsion mode of the main 


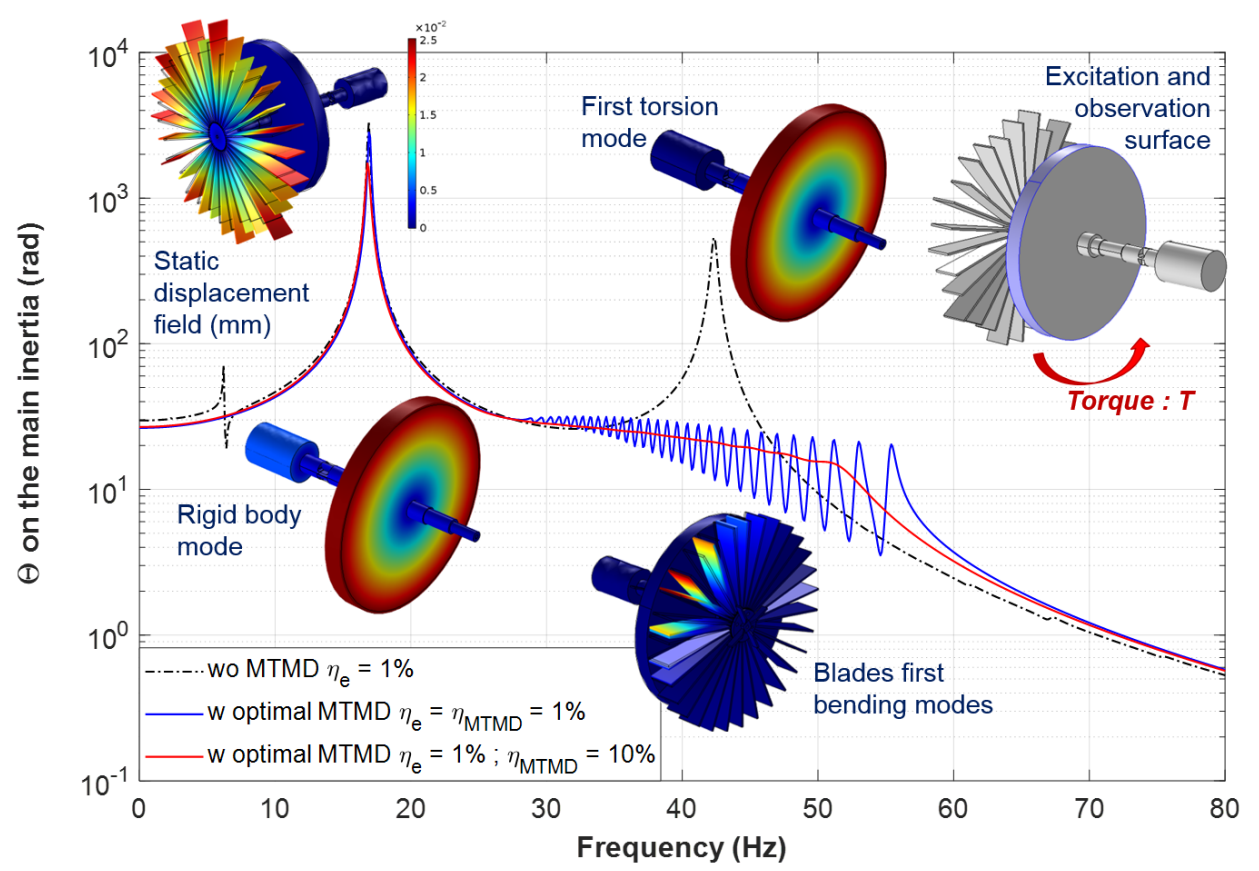

Figure 2: Rotation of the main inertia according to the frequency without and with MTMD at $1500 R P M$

structure. Each blade acts as a dynamic vibration absorber and its natural frequencies are controlled by a set of parameters $\alpha_{i}$. Theses parameters are determined thanks to an optimization method based on the minimization of the mean elastic strain energy on a frequency band around the resonant frequency to control. The proposed methodology can be applied to the case of rotating machines as shown in this paper. Rotating effects such as centrifugal force are taken into account in the finite element model and the use of multi-model basis representative of the stiffness variation around the speed of interest drastically reduce the computation cost of the optimization process. Hence, it is possible to design an optimal MTMD for a given structure and speed as shown in Figure 2.

\section{Acknowledgements}

Authors are grateful to the french company PSA for their financial support.

\section{References}

[1] Masato Abé and Yozo Fujino. Dynamic characterization of multiple tuned mass dampers and some design formulas. Earthquake engineering \& structural dynamics, 23(8):813-835, 1994.

[2] Etienne Balmès. Model reduction for systems with frequency dependent damping properties. Office National d'Etudes et de Recherches Aerospatiales ONERA-Publications-TP, 1997.

[3] A Carcaterra, A Akay, and C Bernardini. Trapping of vibration energy into a set of resonators: Theory and application to aerospace structures. Mechanical Systems and Signal Processing, 26:1-14, 2012.

[4] M Cook. Absolute absorbtion. Car Craft Magazine, 42:75, 1994.

[5] JP Den Hartog. Tuned pendulums as torsional vibration eliminators. Stephen Timoshenko 60th Anniversary Volume, pages 17-26, 1938. 
[6] Hugo Festjens, Chevallier Gaël, Renaud Franck, Dion Jean-Luc, and Lemaire Remy. Effectiveness of multilayer viscoelastic insulators to prevent occurrences of brake squeal: A numerical study. Applied Acoustics, 73(11):11211128, 2012.

[7] Takeru Igusa and $\mathrm{K} \mathrm{Xu}$. Vibration control using multiple tuned mass dampers. Journal of sound and vibration, 175(4):491-503, 1994.

[8] AS Joshi and RS Jangid. Optimum parameters of multiple tuned mass dampers for base-excited damped systems. Journal of sound and vibration, 202(5):657-667, 1997.

[9] Ahsan Kareem and Samuel Kline. Performance of multiple mass dampers under random loading. Journal of structural engineering, 121(2):348-361, 1995.

[10] W Ker Wilson. Practical solution of torsional vibration problems. Chapman and Hall, London, 1968.

[11] Chien-Liang Lee, Yung-Tsang Chen, Lap-Loi Chung, and Yen-Po Wang. Optimal design theories and applications of tuned mass dampers. Engineering structures, 28(1):43-53, 2006.

[12] Hong-Nan Li and Xiang-Lei Ni. Optimization of non-uniformly distributed multiple tuned mass damper. Journal of Sound and Vibration, 308(1-2):80-97, 2007.

[13] Tyler M Nester, Alan G Haddow, Steven W Shaw, John E Brevick, and Victor J Borowski. Vibration reduction in a variable displacement engine using pendulum absorbers. Technical report, SAE Technical Paper, 2003.

[14] Sauvage Olivier. Dispositif de piege vibratoire a resonateurs distribues, 2017. FR Patent 3035939.

[15] BJ Olson, SW Shaw, and C Pierre. Order-tuned vibration absorbers for a rotating flexible structure with cyclic symmetry. In Proceedings of the ASME International Design Engineering Technical Conferences and Computers and Information in Engineering Conference 2005, volume 1, pages 2475-2484, 2005.

[16] Jaewook Park and Dorothy Reed. Analysis of uniformly and linearly distributed mass dampers under harmonic and earthquake excitation. Engineering Structures, 23(7):802-814, 2001.

[17] N Roveri, A Carcaterra, and A Akay. Energy equipartition and frequency distribution in complex attachments. The Journal of the Acoustical Society of America, 126(1):122-128, 2009.

[18] Steven W Shaw and Christophe Pierre. The dynamic response of tuned impact absorbers for rotating flexible structures. Journal of Computational and Nonlinear Dynamics, 1(1):13-24, 2006.

[19] Miller A Wachs. The main rotor bifilar absorber and its effect on helicopter reliability/maintainability. Technical report, SAE Technical Paper, 1973.

[20] Kangming Xu and Takeru Igusa. Dynamic characteristics of multiple substructures with closely spaced frequencies. Earthquake engineering \& structural dynamics, 21(12):1059-1070, 1992.

[21] Hiroki Yamaguchi and Napat Harnpornchai. Fundamental characteristics of multiple tuned mass dampers for suppressing harmonically forced oscillations. Earthquake engineering \& structural dynamics, 22(1):51-62, 1993.

[22] Lei Zuo and Samir A Nayfeh. Minimax optimization of multi-degree-of-freedom tuned-mass dampers. Journal of Sound and Vibration, 272(3-5):893-908, 2004. 